\title{
Assessment of work postures and prevalence of musculoskeletal disorders among porcelain industry workers
}

\author{
Ahmadi A, $\mathrm{MSc}^{1}$, Mirzaei $\mathrm{R}, \mathrm{PhD}^{2 *}$, Ansari $\mathrm{H}, \mathrm{PhD}^{3}$ \\ 1-MSc student, Dept. of Environment Management (HSE), Faculty of Engineering and Technology, Islamic Azad \\ University, Zahedan Branch, Zahedan, Iran. 2-Professor, Dept. of Environmental and Occupational Health \\ Engineering, School of Health, Mashhad University of Medical sciences, Mashhad, Iran. 3- Assistant prof., Health \\ Promotion Research Center, Zahedan University of Medical Sciences, Zahedan, Iran.
}

\begin{abstract}
Received: March 2016, Accepted: May 2016

Background: Today, the prevention of work-related musculoskeletal disorders (MSDs) is considered as a vital issue and one of the highest national priorities. This study was conducted with the aim of evaluating occupational postures and the prevalence of MSDs among porcelain industry workers of the city of Rasht, Iran.

Materials and Methods: This cross-sectional study was done in 2015 on 66 workers, active in different factory units and chosen through stratified random sampling. To determine the frequency of MSDs symptoms in different body parts of the workers, the Nordic Musculoskeletal Questionnaire (NMQ) and Job Content Questionnaire (JCQ) were used. To evaluate the risk of MSDs, the Rapid Entire Body Assessment (REBA) method was utilized. Descriptive statistics, chi-square test, independent $\mathrm{t}$-test, and logistic regression were used for data analysis.

Results: Based on the data obtained through the questionnaires, $36 \%$ of the studied population had experienced pain at least in one body part in the last 12 months. The frequency of pain in the waist, hand and wrists, and neck was the most. According to the data collected through JCQ, the total score of job content had a statistically significant relationship with subjects' age and their occupational background ( $\mathrm{P}<0.001, \mathrm{r}=-0.549$, and $\mathrm{r}=-0.704$, respectively). Mean REBA scores of workers suggested that printing, carpentry, engineering, and material supplement units (scores: 6-6.5) were the most dangerous units, and the enameling unit (score: 4) had the least amount of risk in the porcelain industry.

Conclusions: The present study indicated that, in the studied industry, work posture correction with the help of correction stations and appropriate training is absolutely necessary.
\end{abstract}

Keywords: Assessment, Musculoskeletal, Disorders, Porcelain, Industry, Questionnaire, Job

\section{Introduction}

Musculoskeletal disorders (MSDs) are the major causes for workers' inability, expense raise, and efficiency reduction in industrialized and developed countries. Contrary to many work-related diseases, whose source is contact with a certain dangerous substance/material, MSDs are multifactorial and have various psychological, social, and individual causes in addition to physical causes.

Force, posture, recurrent and repetitive movements, and work duration are important physical risk factors in the emergence of MSDs. In 2010, Shaou et al. performed a study on MSDs among construction workers in the western states of Bengal, India, using Rapid Entire Body Assessment (REBA) and the Nordic Musculoskeletal Questionnaire (NMQ) (1). They found that the practical stages of REBA were stage 3 and stage 4 , and the frequency of pain was high in different body parts of the workers, such as the waist. They also reported that by training workers on the correct form of load lifting, predicting break

\footnotetext{
* Corresponding author: Ramazan Mirzaei, Dept. of Environmental and Occupational Health Engineering, School of Health, Mashhad University of Medical sciences, Mashhad, Iran Email: rammir277@gmail.com
} 
time, correcting work methods, and designing ergonomic facilities, MSDs among construction workers would be prevented (1).

Maulik and Iqbal conducted a research on MSDs among medical laboratory technicians of a university in India using the NMQ and Quick Exposure Check (QEC) method (2). Their results showed that $66.9 \%$ of technicians had been suffering from MSDs for 12 months and the frequency of pain in the waist (44.0\%), knees (20.7\%), and neck (18.4\%) was the highest in these subjects. They concluded that there was a high or very high risk of MSDs among $85.3 \%$ of laboratory technicians. Moreover, this study showed a statistically significant relationship between the risk level and the prevalence of MSDs among technicians $(\mathrm{P}<0.05)$, and most of the observed risk factors were inappropriate standing postures and work stations (2).

Maulik et al. also conducted a study on work postures and prevalence of MSD symptoms among medical technicians using the NMQ and Rapid Upper Limb Assessment (RULA) (3). Their findings indicated that MSDs were more common among $73.3 \%$ of technicians, and pain locations were mostly the trunk, knees, neck, and ankles. Additionally, the statistical results demonstrated that there was a significant relationship between the MSD scores before and after work (3).

Moreover, in 2004, in a study by Punnett et al., checkups on 8200 workers showed that the observed symptoms in distal MSDs had a relationship with stressful ergonomic factors and job content. They were also related to the gained scores (4).

Considering the literature, posture analysis is an efficient way of evaluating work activities, and posture or body position as a source of MSDs has attracted attention. Posture analysis is a suitable method for evaluating activities which are done while standing and are performed with pressured upper or lower body parts.

This study was conducted with the objective to determine work postures and prevalence of MSDs among porcelain industry workers. It is hoped that, with the help of this study, the attention of the related authorities is drawn to the side effects and hazards of MSDs and the evaluation of ergonomic risk factors of occupations in the porcelain industry. It is also hoped that this will result in the implementation of the required measures to provide the standards of a work environment, execution of safety and health regulations, maintenance of workers' health, increased production and product quality, and society's economic growth.

\section{Material and Methods}

The participants of this cross-sectional study were selected through stratified random sampling from among porcelain industry workers of the city of Rasht, Iran, in 2015. The participants were divided into two groups of subjects with and without pain. Some demographic variables of the workers, such as age and education level, and discomfort were studied. Sample size was calculated based on the sample size formula with $\alpha$ equal to $10 \%$, approximate prevalence of $75 \%$, and accuracy of $5 \%$. The height and weight of the subjects were measured using a tape meter (accuracy $\mathrm{mm}$ ) and scales (accuracy $100 \mathrm{mg}$ ), respectively, and body mass indexes (BMI) were calculated. The studied postures were evaluated through REBA method by observing and filming the body positions of the personnel in critical postures while performing their tasks. Subsequently, the data were inserted into the analysis software of REBA (NexGen Ergonomics Inc., Canada). The REBA method is a rapid and easy observational postural analysis tool for whole body activities and evaluates the ergonomics risk factor by directly observing the posture of employees while they are working at their workstation. The posture of each body organ is given a score, and then, posture scores are determined based on the diagram presented in figure 1. Accordingly, scores A and B are taken from the standard tables and loading/forced coupling scores are determined 
based on the load of the instrument on the hands during work time (load $<11 \mathrm{lbs} .=0$, 11-22 lbs. = 1, and so on). Then, loading score is added to scores $\mathrm{A}$ and $\mathrm{B}$, and finally, REBA score is obtained and the risk level is determined based on table 1 for corrective action decision (5).

The data related to the prevalence of MSDs in the 49 weeks was collected using the NMQ and Job Content Questionnaire (JCQ) based on psychological and psychological-occupational requirements. Using a questionnaire containing 20 items, physical and mental occupational requirements can be evaluated in terms of workers; and the impact of mental and subjective factors on the prevalence of MSDs can be examined. The validity of the questionnaire was approved with at least 0.83 content validity index (CVI) for every question by 8 experts. The reliability of the NMQ and JCQ was approved using Cronbach's alpha ( $\alpha$ $=0.72$ and 0.62 , respectively). Workers who had a congenital / inborn disorder or had undergone surgery due to non-occupational disorders were not considered into account.

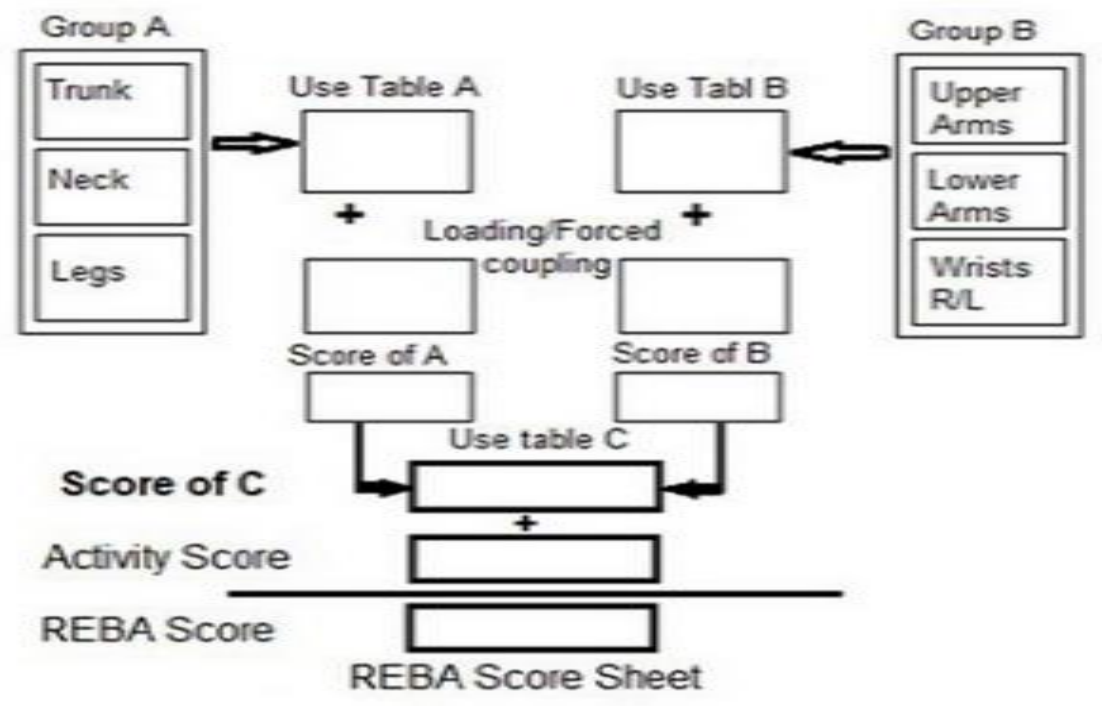

Figure 1: Rapid Entire Body Assessment (REBA) score sheet

REBA was utilized to evaluate work postures (inappropriate position of body parts while working), which enables us to analyze the position of body parts during work and evaluates the ergonomic condition of the occupation. In REBA, different work stages were filmed in order to identify the postures. Next, the studied postures were chosen to include at least one or several samples of each class, ergonomically highly risky or very damaging to the musculoskeletal system. Then, each of the 9 joints in the chosen postures was closely examined. After encoding the data using SPSS software (version 20, SPSS Inc., Chicago, IL, USA), they were analyzed using Spearman correlation test. For data description, descriptive statistics, including central and dispersion indexes and statistical charts and graphs, were used. For data analysis, in order to determine the relationship among all qualitative variables, chi-square test, and to determine the relationship between qualitative and quantitative variables, independent t-test or ANOVA were used. Multiple logistic regression was used to investigate the effect of predictive factors on pain among workers by controlling potential confounders. All $\mathrm{P}$ values of less than 0.05 were considered significant. 
Table 1: Rapid Entire Body Assessment (REBA) decision

\begin{tabular}{ccc}
\hline REBA score & Risk level & Action level \\
\hline $2-3$ & Low & Corrective action including further assessment may be necessary \\
\hline $4-7$ & Average & Corrective action including further assessment is necessary \\
\hline $8-10$ & High & Corrective action including further assessment is necessary soon \\
\hline $11-15$ & Very high & Corrective action including further assessment is necessary now \\
\hline
\end{tabular}

\section{Results}

In this study, 66 male workers with a mean age of $35.7 \pm 7.1$ years, weight of $82.6 \pm 12.4 \mathrm{Kg}$, height of $172 \pm 14.2 \mathrm{~cm}$, BMI of $28.2 \pm 6$, and job experience of $11.2 \pm 6.6$ years were studied. As illustrated in table 2, the most frequent pain and disorder location in the last 12 months among the workers was the waist. In this study, 17 workers $(25.8 \%)$ had no problem in any body parts. Moreover, 40 workers $(60.6 \%)$ had diploma, and 18 of them $(27.3 \%)$ had an education degree less than diploma and 8 of them (12.1\%) were higher than diploma.

Based on the results of data analysis and chisquare test, there was no statistically significant relationship between hand dominance (left-handedness, righthandedness) and pain, discomfort, and numbness in the last 12 months $(P=0.492)$. A statistically significant difference was observed between the average job backgrounds of the two groups of "with pain" and "without pain" $(\mathrm{P}<0.001)$; the longer is the work experience, the greater is the pain. There were no significant differences between the average BMI of the two aforementioned groups $(\mathrm{P}=0.071)$. Furthermore, Fisher's exact test displayed a significant difference between the education levels of these two groups in the last 12 months $(\mathrm{P}=0.004)$. Thus, it is concluded that, more educated people had less pain and MSDs.

Table 2: Pain, discomfort, and numbness distribution during the last 12 months based on the studied organ

\begin{tabular}{ccc}
\hline \multirow{2}{*}{ The 9 body parts } & \multicolumn{2}{c}{ Frequency } \\
\cline { 2 - 3 } & Number & Percentage \\
\hline Neck & 34 & 51.5 \\
\hline Shoulders & 31 & 47.0 \\
\hline Elbows & 28 & 42.4 \\
\hline Hands and wrists & 35 & 53.0 \\
\hline Back & 9 & 13.6 \\
\hline Waist & 38 & 57.6 \\
\hline Buttocks and thighs & 1 & 1.5 \\
\hline Knees & 23 & 34.8 \\
\hline Legs and ankles & 15 & 22.7
\end{tabular}

In reviewing distribution indexes of job content condition, as indicated in table 2 and figure 1, there was a significant and reverse relationship between total JCQ score and the subjects' ages $(\mathrm{P}<0.001$ and $r=-0.549$ ). Furthermore, there was a statistically significant relationship between total JCQ score and the occupational background of the participants $(\mathrm{P}<0.001$ and $\mathrm{r}=-0.704)$. However, the results of ANOVA showed no significant difference between average scores of physical occupational requirements $(P=0.096)$, psychological occupational requirements $(P=0.161)$, and job content operation $(\mathrm{P}=0.120)$ at various educational levels (Figure 2$)$. 


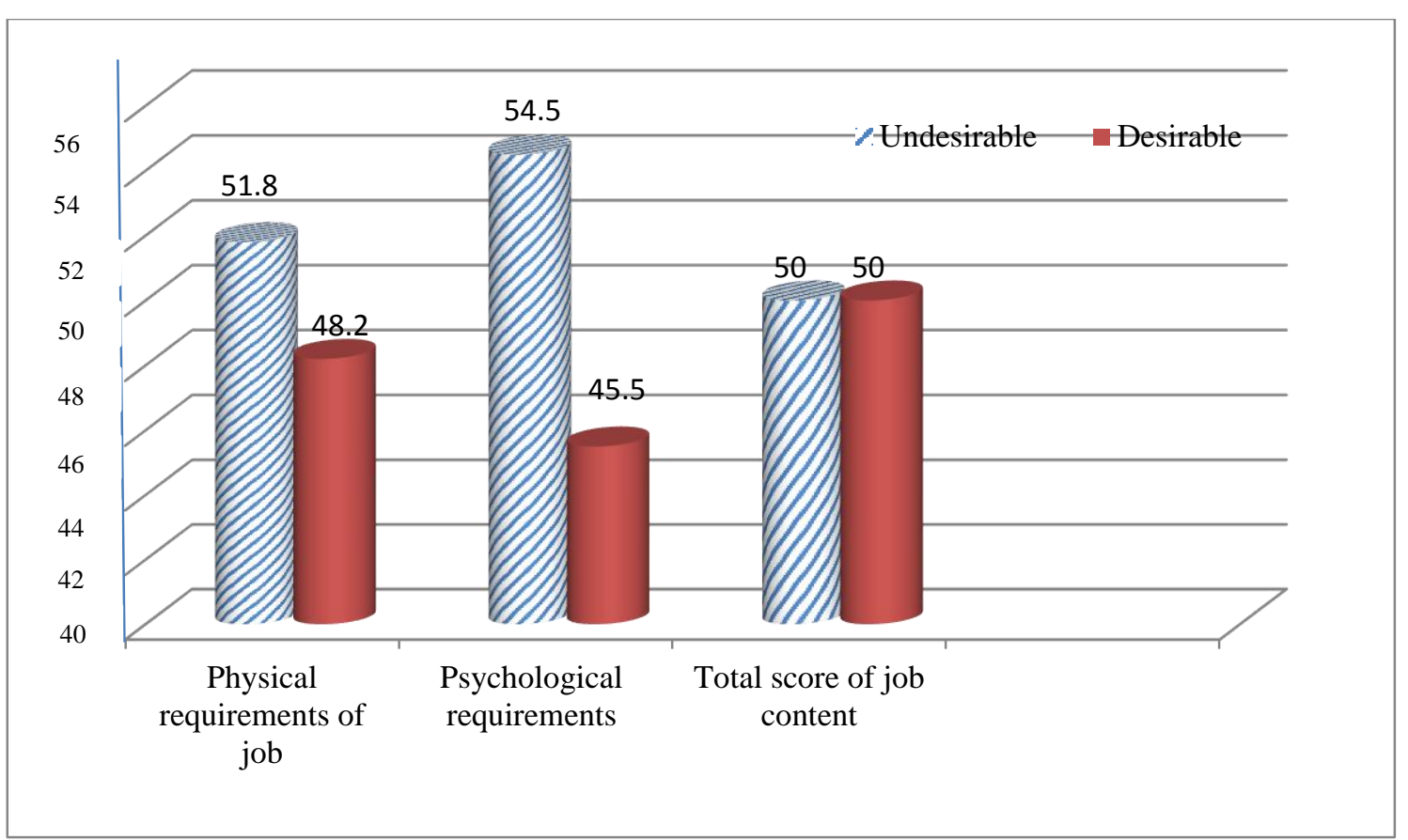

Figure 2: Categorization of the studied workers based on job content scores

As observed in table 3, the majority of the workers obtained an average or high REBA score, and $86.4 \%$ of them needed to practice corrective measures. There was no statistically significant relationship between REBA score and BMI, age, and job experience of the workers, and the observed correlation was not noticeable $(\mathrm{P}>0.05)$.

Table 3: Risk level distribution among porcelain industry workers based on Rapid Entire Body Assessment (REBA)

\begin{tabular}{llll}
\hline Risk level & REBA score & Number & Percentage \\
\hline Low & $2-3$ & 9 & 13.6 \\
\hline Average & $4-7$ & 42 & 63.6 \\
\hline High & $8-10$ & 14 & 21.2 \\
\hline Very high & $11-15$ & 1 & 1.5 \\
\hline
\end{tabular}

As shown in table 4 , based on the results of logistic regression job background, education, and BMI had no statistically significant relationship with pain in the last 12 months. Nevertheless, there was a statistically significant relationship between age and pain in the last 12 months $(\mathrm{P}<0.04)$. These variables altogether considerably predict pain and disorder in the last 12 months $\left(\mathrm{R}^{2}=0.4\right)$. Among independent variables, the variables of education (reverse relationship) and job experience have displayed the highest amount of tendency.

Table 4: Logistic regression components and related amounts of pain and discomfort in body parts in the last 12 months

\begin{tabular}{lllll}
\hline Variable & P-value & Wald statistic & Standard error & Intercept (B spot) \\
\hline Body mass index & 0.288 & 1.130 & 0.058 & 0.061 \\
\hline Age & 0.604 & 0.269 & 0.077 & 0.040 \\
\hline Job background & 0.019 & 5.498 & 0.131 & 0.307 \\
\hline Education & 0.164 & 1.934 & 0.340 & -0.472 \\
\hline
\end{tabular}




\section{Discussion}

Based on data analysis, ergonomic risk level, which was measured through REBA method, was not suitable among the personnel of Rasht Porcelain Company, Iran. In addition, the frequency of pain in different body parts (waist, hands and wrists, neck, shoulders, elbows, and etc.) based on the NMQ among the personnel of Rasht Porcelain Company was high. Based on the JCQ, psychological and mental occupational factors influencing the prevalence of MSDs were inappropriate in the studied subjects.

As the results of the present study indicate, most cases of discomfort of body parts due to problems such as pain, discomfort, and numbness were related to the waist. This finding was in agreement with that of a study by Mohammadfam et al. (6). Their research assessed the risk of MSDs with the use of Postural Loading on the Upper Body Assessment (LUBA) and QEC method among the employees of an industrial company (6).

The results of this study showed a statistically significant correlation between average amounts of age and pain in the last 12 months ( $\mathrm{P}<0.04, \mathrm{P}<0.05$, respectively). Sharafi et al. have come to a similar conclusion about the correlation between the variable of age and the frequency of MSDs among the carpet weavers of Sanandaj City, Iran (7).

In this study, no significant relationship was found between the two types of hand dominance (left-handedness and righthandedness) and pain and discomfort. This finding was in accordance with that of the study by Mehrparvar et al.(8), who assessed the risk of MSDs based on QEC method at a food production factory. In addition, this finding is in agreement with the results of the study by Choobineh et al. at a tire factory (8). Frequency percentage of pain, discomfort, and numbness in the 9 studied body parts during a 12-month-long survey was, respectively, higher in the waist, hands and wrists, neck, and shoulder. This finding is in agreement with the findings of the study by Nogueria et al. on the indicators of psychological disorders/ problems and MSDs among the workers of the repair and maintenance unit of an airline using NMQ and JCQ (9).

In this study, distribution of musculoskeletal problems, which inhibited daily life activities during the last 49 weeks, was mostly related to the neck, shoulders, hands and wrists, and waist. This finding was in accordance with the findings of Alrowayeh et al. (10). They performed a study on a group of rehabilitation specialists in Kuwait with the use of the NMQ in order to obtain the frequency of MSDs among this group. In their study, the most important MSDs were reported to be in the waist, wrists, knees, feet, and ankles (10).

In this study, the statistical distribution of job content was found to have a significant relationship with the body parts in risk of pain, discomfort, and numbness $(\mathrm{P}<0.05)$. This finding was in agreement with the findings of the study by Nogueria et al. (9).

On the basis of statistical distribution, investigation of the level of danger among the workers through REBA method illustrated that more than half of them were at the average risk level (63.6\%). These workers and the workers of above-mentioned groups (people "with pain" and people "without pain") require corrective actions. This finding was in agreement with the findings of the study by Sumita et al. on the prevalence of MSDs among Indian dentists based on REBA method. They found that the REBA score was high and work postures must be corrected (11). Between REBA score and the workers' BMI, no significant statistical relationship was found and the observed correlation was not noticeably important. This finding (because of kind of work and study population) stands in contrast to the finding of the study by Sharafi et al. on the prevalence of MSDs and its relationship to work postures based on QEC method. They showed that increased BMI is the most important reason for the high frequency of disorders in the waist area (7).

Based on regression results, the work experience variable was an appropriate 
predictor of work-related injuries during the research period. The variables of work experience and BMI, respectively, are good predictors of absence from work due to workrelated injuries.

With the use of regression test, no significant statistical relationship was found among the variables of age, work experience, education, and BMI and REBA score. This finding is in accordance with the results of the study by Varmaziar et al. on work postures of medical packaging workers with the use of the RULA method and Body Map Questionnaire (10). They reported that there was no correlation between the total RULA score and age, weight, and work experience.

Suggestions in this research include:

1. Due to inappropriate postures (poor range of motion, load lifting, use of non-ergonomic work tools, and inappropriate work level) in industries, it is essential to design an appropriate standard work environment based on ergonomics, safety, and occupational health.

2. In order to obviate the physical and psychological needs of the employees of the surveyed industry, it is recommended to reduce the physical and mental burden on the worker and provide appropriate social welfare services for them.

3. Based on the REBA method, most of the investigated workers were at an occupational risk level that required corrective measures; therefore, their jobs must be prioritized in ergonomics intervention programs.

4. Taking into account the frequency of workrelated risk factors in the process of identifying and evaluating the work environment of the studied industries, to control risk factors, especially for decreasing and removing inappropriate postures, the use of a three-level pyramid of control in order of preference is suggested for engineering, administrative/ executive, and training tasks/ measures.

5. The results of the present research illustrated that employees with more work experience were at greater risk of MSDs.

\section{Conclusion}

Evaluation of work postures and the prevalence of MSDs among the personnel of Rasht Porcelain Company showed that MSDs among the employees were mostly reported in the neck, hands and wrists, waist, and knees. Disorders among employees were due to the nature of their jobs. According to the REBA scores, the most important reasons for these disorders were inappropriate postures (limited range of motion, incorrect load carrying, use of non-ergonomic work tools, and improper work level). The level of physical and psychological needs of these workers is high, which increases the probability of physical injuries such as MSDs. In addition, REBA results showed that most of the participants of this study are in need of corrective actions. This suggests that the employees' work environment is not ergonomically suitable.

\section{Acknowledgments}

This research was conducted with the help of the professors of the School of Engineering of Islamic Azad University. We appreciate the efforts of all those who have cooperated in conducting this study, especially Dr. Mohammad Imani.

Conflict of interests: None declared.

\section{Reference}

1. Sahu S, Chattopadhyay S, Basu k, Paul G. The ergonomic evaluation of work-related musculoskeletal disorders among construction labourers working in unorganized sectors in West Bengal, India. J Hum Ergol 2010; 39(2):99-109.

2. Maulik Sh, Iqbal R. Occupational health and musculoskeletal symptoms among Indian Medical Laboratory technicians. Journal of Occupational Health and Epidemiology 2013; 2(3):82-92.

3. Maulik S, Iqbal R, De A, Chandra AM. Evaluation of the working Posture and prevalence of musculoskeletal symptoms 
among medical laboratory technicians. J Back Musculoskelet Rehabil 2014; 27(4):453-61.

4. Punnett L, Gold J, Katz JN, Gore R, Wegman DH. Ergonomic stressors and upper extremity musculoskeletal disorders in automobile manufacturing: a one year fallow up study. Occup Environ Med 2004; 61(8):668-74.

5. Shizaei M, Mirzaei R, Khaje-Alizade A, Mohammadi M. Evaluation of ergonomic factors and postures that cause muscle pains in dentistry students' bodies. J Clin EXP Dent 2015; 7(3):e414-8.

6. Mohammadfam I, Kianfar A, Afsartala B. Assessment of musculoskeletal disorders in a Manufacturing company using QEC and LUBA methods and comparison of results. Iran Occupational Health 2010; 7(1):54-60.

7. Sharafi N, Gharibi F, Khoobi J. Prevalence of musculoskeletal disorders and its relation to working posture in Sanandaj hand-woven carpet weavers. Scientific Journal of Kurdistan University of Medical Sciences 2014; 19(4):19.

8. Mehrparvar AH, Ranjbar S, Mostaghaci M, Salehi M. Risk assessment of musculoskeletal disorders by QEC method in a food production factory. Journal of Safety Promotion and Injury
Prevention 2011; 3(2):54-60

9. Nogueria HC, Diniz AC, Barbieri DF, Padula RS, Carregaro RL, de Oliveira AB. Muscul Skeltal disorders and psychosocial risk factors among workers of the aircraft maintenance industry. Work 2012; 41(Suppl 1):4801-7.

10. Alrowayeh HN, Alshatti TA, Aljadi SH, Fares M, Alshamire MM, Alwazan SS. Prevalence, characteristic, and impacts of work-related musculoskeletal disorders: a survey among physical therapists in the state of Kuwait. BMC Musculoskelet Disord 2010; 11:116. Doi:10.1186/1471-2474-11-116.

11. Sumita B, Sameeksha H, Lalitagauri M. Prevalence of musculoskeletal disorders among indian dentists: a pilot survey with assessment by rapid entire body assessment. World Journal of Dentistry 2015; 6(1):39-44.

12. Varmaziar S, Safari A, Younesi M. Assessment of working postures and prevalence of musculoskeletal disorders in between staff of the packing of medicines by using RULA method and Body map questionnaire. Paper presented at: The first international conference on ergonomics Iran; 2010 May 7-8; Tehran, Iran. 